\title{
Determining the role of adjuvant radiotherapy in the management of meningioma: a Surveillance, Epidemiology, and End Results analysis
}

\author{
*Abhinav K. Reddy, MS, James S. Ryoo, BS, Steven Denyer, MS, Laura S. McGuire, MD, and \\ Ankit I. Mehta, MD
}

Department of Neurosurgery, University of Illinois at Chicago, Illinois

\begin{abstract}
OBJECTIVE The aim of this study was to illustrate the demographic characteristics of meningioma patients and observe the effect of adjuvant radiation therapy on survival by using the Surveillance, Epidemiology, and End Results (SEER) database. More specifically, the authors aimed to answer the question of whether adjuvant radiotherapy following resection of atypical meningioma confers a cause-specific survival benefit. Additionally, they attempted to add to previous characterizations of the epidemiology of primary meningiomas and assess the effectiveness of the standard of care for benign and anaplastic meningiomas. They also sought to characterize the efficacy of various treatment options in atypical and anaplastic meningiomas separately since nearly all other analyses have grouped these two together despite varying treatment regimens for these behavior categories.
\end{abstract}

METHODS SEER data from 1973 to 2015 were queried using appropriate ICD-O-3 codes for benign, atypical, and anaplastic meningiomas. Patient demographics, tumor characteristics, and treatment choices were analyzed. The effects of treatment were examined using a multivariate Cox proportional hazards model and Kaplan-Meier survival analysis.

RESULTS A total of 57,998 patients were included in the analysis of demographic, meningioma, and treatment characteristics. Among this population, cases of unspecified WHO tumor grade were excluded in the multivariate analysis, leaving a total of 12,931 patients to examine outcomes among treatment paradigms. In benign meningiomas, gross-total resection (HR 0.289, $p=0.013$ ) imparted a significant cause-specific survival benefit over no treatment. In anaplastic meningioma cases, adjuvant radiotherapy imparted a significant survival benefit following both subtotal (HR 0.089, $p=$ 0.018 ) and gross-total (HR 0.162, $p=0.002$ ) resection as compared to gross-total resection alone. In atypical tumors, gross-total resection plus radiotherapy did not significantly change the hazard risk (HR 1.353, $p=0.628$ ) compared to gross-total resection alone. Similarly, it was found that adjuvant radiation did not significantly benefit survival after a subtotal resection (HR 1.440, $p=0.644)$.

CONCLUSIONS The results of this study demonstrate that the role of adjuvant radiotherapy, especially after the resection of atypical meningioma, remains somewhat unclear. Thus, given these results, prospective randomized clinical studies are warranted to provide clear information on the effects of adjuvant radiation in meningioma treatment.

https://thejns.org/doi/abs/10.3171/2019.3.FOCUS1971

KEYWORDS meningioma; radiotherapy; survival; tumor management; SEER; surgery

$\mathrm{M}$ ENINGIOMAS are one of the most prevalent forms of tumor seen in the central nervous system (CNS), accounting for about a third of all primary brain and CNS tumors. ${ }^{15}$ They typically arise from arachnoid cap cells in the meninges surrounding the brain and spine, can be readily diagnosed with imaging, and are often found incidentally during imaging for other medical purposes. ${ }^{21}$ An increase in cranial imaging for a variety of medical indications has led to a greater reported incidence of primary meningioma in patients who were not originally being assessed for meningioma. ${ }^{18}$ The majority of meningiomas are benign, slow-growing tumors (ICD-O-3 behavior 0); however, some forms are more aggressive and are categorized as either atypical (ICD-O-3 behavior 1) or anaplastic (ICD-O-3 behavior 3) meningiomas. ${ }^{1}$

Previous epidemiological studies have shown that be-

ABBREVIATIONS CNS = central nervous system; GTR = gross-total resection; NF2 = neurofibromatosis type 2; NOS = not otherwise specified; SEER = Surveillance, Epidemiology, and End Results; STR = subtotal resection.

SUBMITTED February 1, 2019. ACCEPTED March 25, 2019.

INCLUDE WHEN CITING DOI: 10.3171/2019.3.FOCUS1971.

${ }^{*}$ A.K.R. and J.S.R. contributed equally to this paper. 
nign meningiomas represent approximately $94 \%$ of all meningioma cases, whereas atypical and anaplastic meningiomas account for about $4 \%$ and $1 \%$, respectively. ${ }^{1}$ These tumors are most common in older individuals, with an average age of approximately 64 years at onset and an increasing incidence with age. ${ }^{1}$ There is also a well-documented increased incidence of these tumors in women (73\% in women overall ${ }^{8}$ and $80 \%$ of spinal cord meningiomas in women ${ }^{23}$ ). Some evidence suggests that this trend is not seen in pediatric patients, ${ }^{8}$ in whom these tumor types are rare and likely associated with neurofibromatosis type 2 (NF2) or therapeutic radiation for other malignancies. ${ }^{11}$ Overall, death from benign meningiomas is very low with exceptions for tumors in locations such as the skull base where the risk from tumor growth is greater. ${ }^{5}$ Atypical and anaplastic meningiomas have lower survival rates and are often associated with recurrence. ${ }^{10}$

Given that benign meningiomas are a slow-growing malignancy, the usual treatment course involves observation after diagnosis through imaging. ${ }^{24}$ For most patients, the meningioma will be asymptomatic, and as long as the tumor size does not drastically increase, there is no urgency to treat surgically. ${ }^{4}$ However, an exception to this stance relates to pediatric patients, in whom there is the concern that future surgery will be required and the decision to perform gross-total resection (GTR) early may be made. ${ }^{14}$ For most benign meningiomas for which GTR is successfully completed, adjuvant radiotherapy is not indicated. ${ }^{5}$ For atypical meningiomas, which have a higher rate of recurrence, standard practice is to perform GTR when possible; however, there is ongoing debate over whether adjuvant radiotherapy should be provided in cases in which GTR is successful, with studies showing various effects on survival. ${ }^{2,3,19}$ The advent of newer, more focused radiotherapy options has added to the question of whether to radiate in these cases and whether that radiation will improve outcomes. For anaplastic meningiomas, which are highly aggressive and come with a poor prognosis, GTR and subsequent radiotherapy are both indicated..$^{16}$

In the present study, in which we conducted the largest-to-date Surveillance, Epidemiology, and End Results (SEER) analysis of primary meningiomas, we aimed to answer the question of whether adjuvant radiotherapy following resection of atypical meningioma confers a causespecific survival benefit. Additionally, we attempted to add to previous characterizations of the epidemiology of primary meningiomas and assess the effectiveness of the standard of care for benign and anaplastic meningiomas. We also sought to characterize the efficacy of various treatment options in atypical and anaplastic meningiomas separately since nearly all other analyses have grouped these two together despite varying treatment regimens for these behavior categories.

\section{Methods \\ Study Sample}

A retrospective analysis was performed using the SEER Program database collected by the National Cancer Institute from 1973 to 2015. The data set entitled "Incidence - SEER 18 Regs Custom Data (with additional treat- ment fields), Nov2017 Sub (1973-2015 varying) - Linked To County Attributes - Total U.S., 1969-2016 Counties" was used, and SEER*STAT version 8.1.2 was used to extract case-level data. SEER contains de-identified individual-level data from 13 cancer registries across the United States.

The ICD-O-3 codes were used to identify 89,525 cases of CNS meningioma for the years 1973-2015. Those cases with data collected from autopsy or death certificate and records with incomplete cause-specific survival data were excluded from our study. ICD-O-3 codes with more than 30 patients and records with only one tumor recorded were included in the study, which resulted in a total of 57,998 cases.

\section{Baseline Characteristics}

Analyzed patient demographics included age group (0-19, 20-39, 40-59, 60-79, and 80+ years), sex (female, male), race (black, white, other), and marital status (married/domestic partner, divorced/separated, single, widowed). Studied tumor characteristics included primary tumor site (cerebral meninges C700, spinal meninges C701, meninges not otherwise specified [NOS] C709, brain C710-C719, spinal cord C720, cauda equina C721, olfactory nerve $\mathrm{C} 722$, optic nerve $\mathrm{C} 723$, acoustic nerve C724, cranial nerve NOS C725, overlapping lesion of brain and CNS C728, nervous system NOS C729, pituitary gland C751, craniopharyngeal duct C752, pineal gland C753) and histology (9530/0: meningioma, NOS; 9531/0: meningothelial meningioma; 9532/0: fibrous meningioma; 9533/0: psammomatous meningioma; 9534/0: angiomatous meningioma; 9537/0: transitional meningioma; 9530/1: meningiomatosis, NOS; 9538/1: clear cell meningioma; 9539/1: atypical meningioma; 9530/3: meningioma, anaplastic; 9533/3: psammomatous meningioma, anaplastic). Treatment characteristics included biopsy, resection (subtotal resection [STR]; GTR, NOS), radiation (yes, no), and chemotherapy (yes, no).

\section{Statistical Analysis}

CNS meningiomas were grouped according to behavior category ( 0 , benign; 1 , atypical; 3 , anaplastic). Patient demographics, tumor characteristics, and treatment regimens for the records identified are summarized in Tables 1 and 2. The effect of treatment regimen on patient survival was evaluated using a Cox proportional hazards model including age, race, sex, marital status, tumor size, histological type, anatomical location, and WHO grade (Tables 3-6). A Firth bias correction was applied to account for the small sample sizes seen in some subgroups. ${ }^{12}$ Mortality was defined as cause-specific mortality from a CNS cancer and did not count individuals who died of noncancerous causes, with the follow-up from date of diagnosis until 2015. Additionally, graphic analysis using the Kaplan-Meier method was fitted to evaluate the association between treatment sequence and survival, stratified by anatomical location. Multiple comparisons for the log-rank test with Sidak correction were then performed to compare the different treatment regimens. Multivariate logistic regression models adjusted for sex, age groups, 


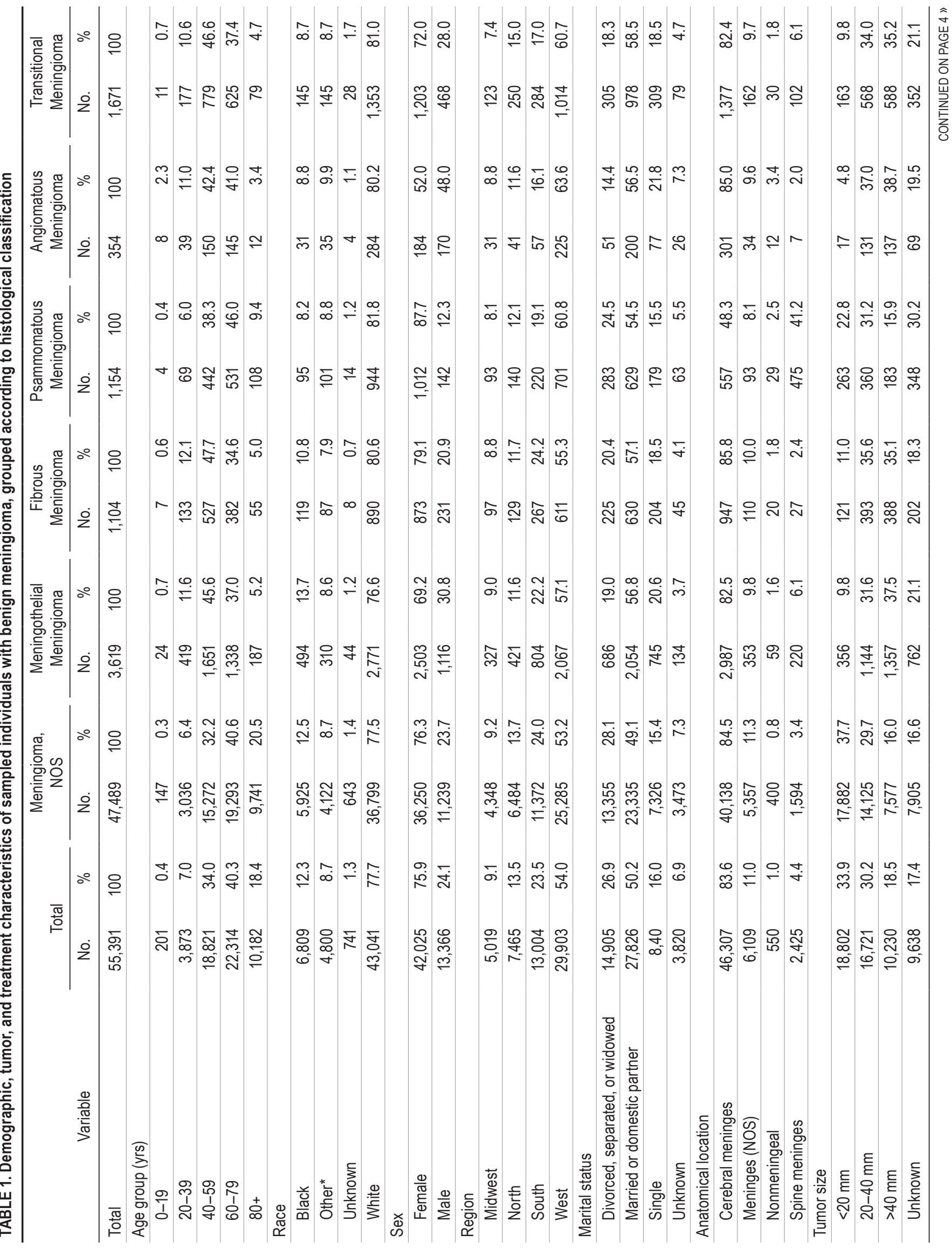




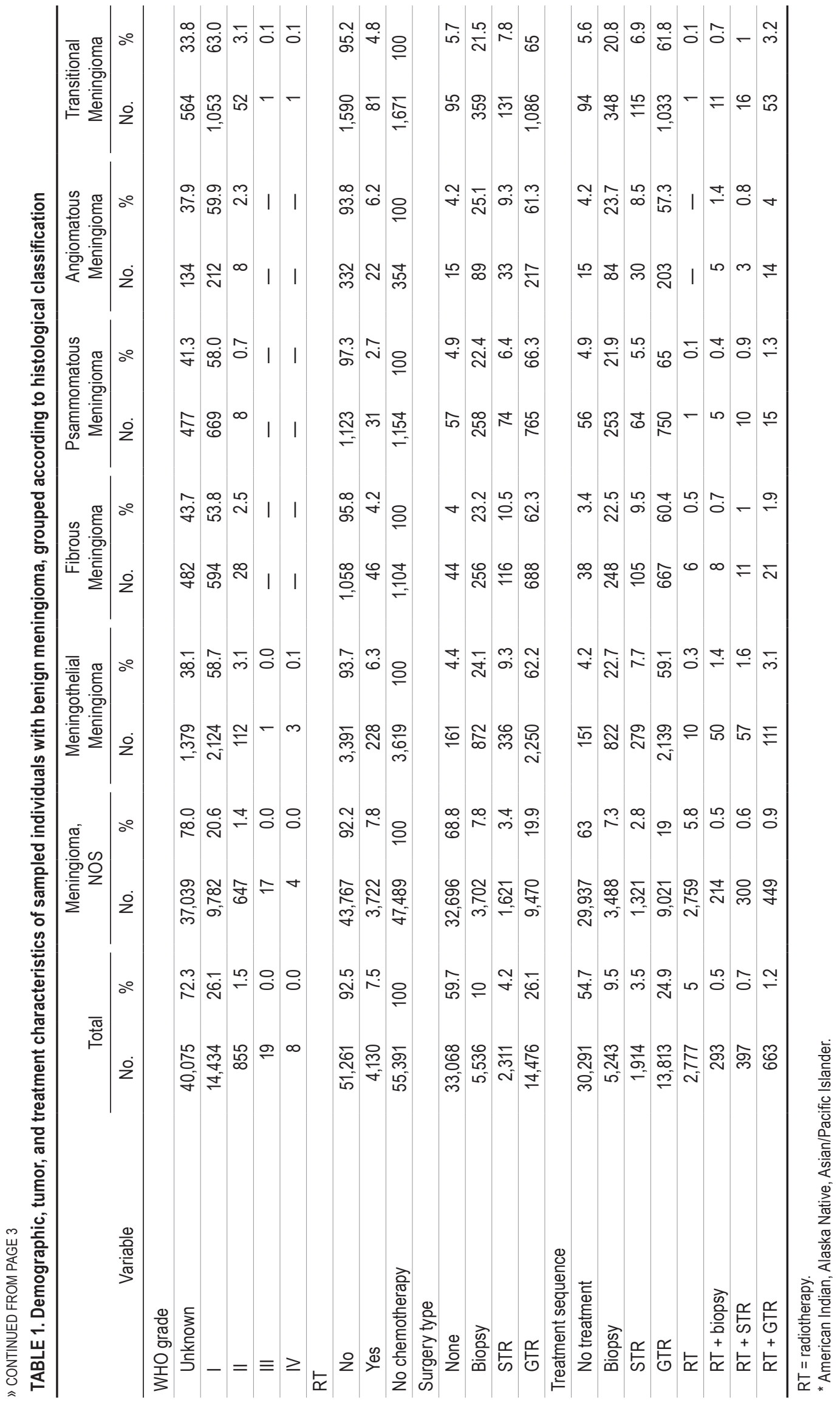




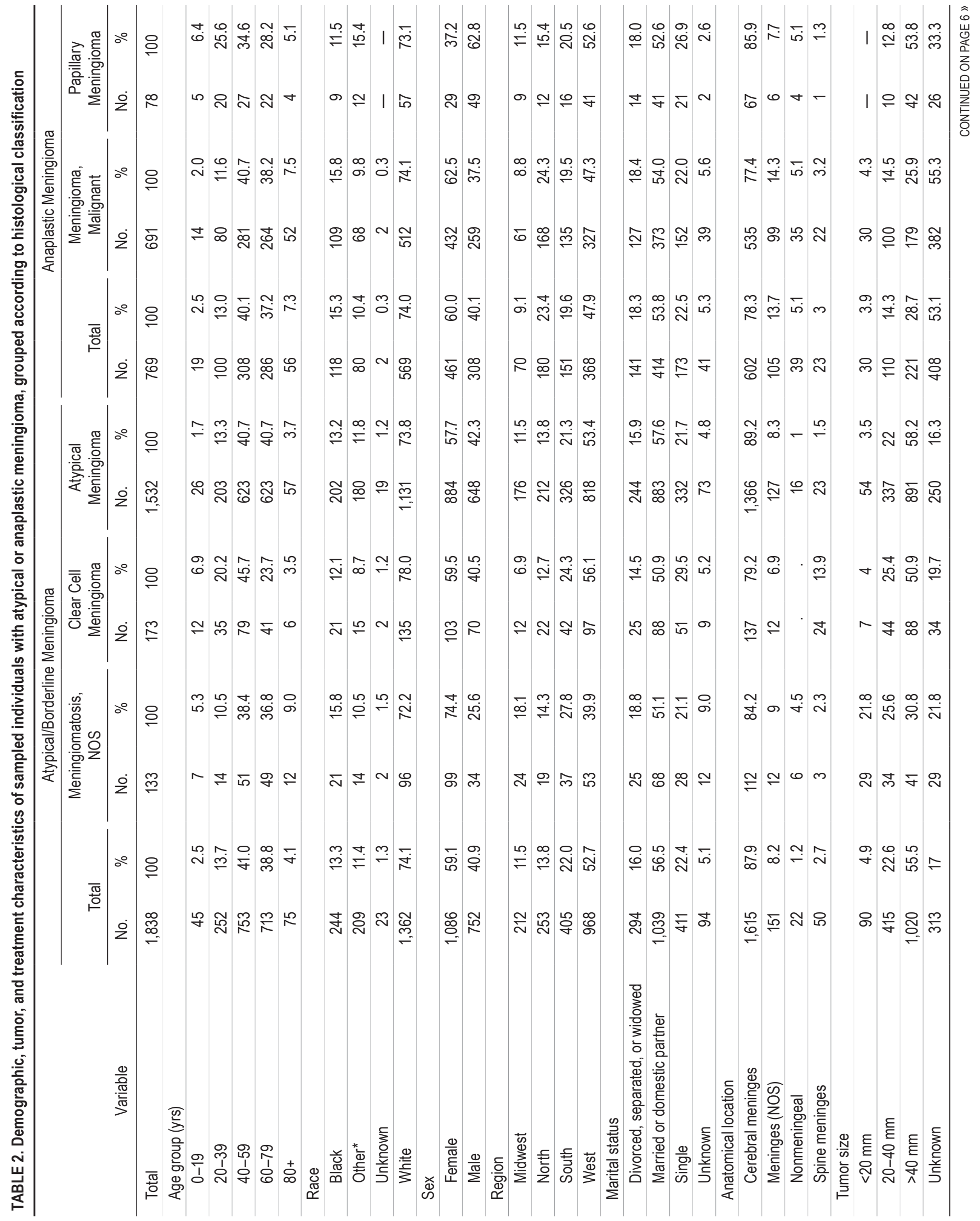




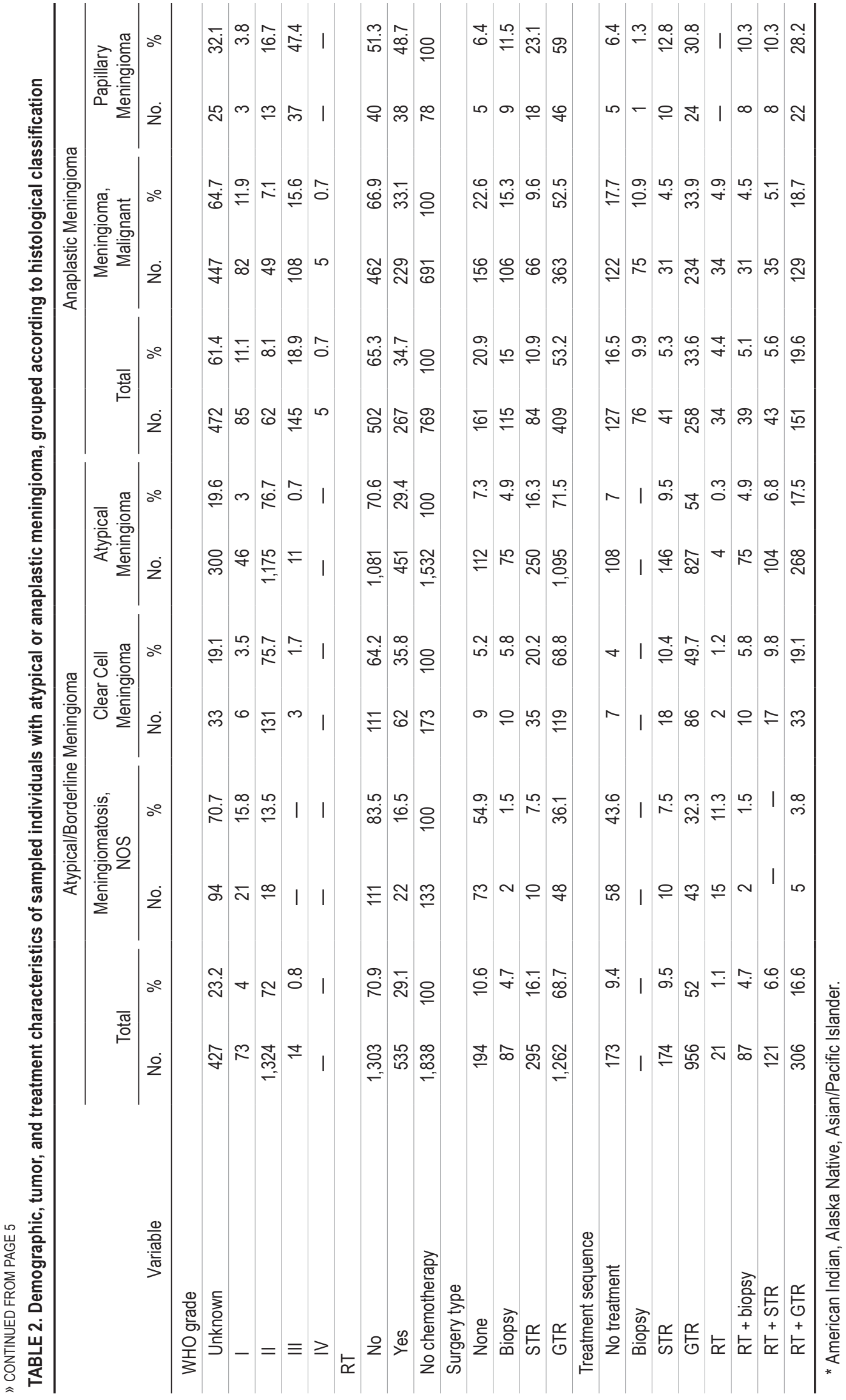


race, marital status, histological type, anatomical location, tumor size, WHO grade, radiation, and surgical intervention were fitted to evaluate the association between treatment sequence and cause-specific survival (Fig. 1). Only variables with more than $80 \%$ completion rates were included in the multivariate model. For the survival analyses mentioned above, only samples for which the WHO grade was recorded were included in the analysis. Additionally, since the WHO only recognizes grades I, II, and III as options in the grading of meningiomas, patients with a reported WHO grade IV tumor were removed from all survival analyses. The significance level was set at alpha = 0.05 . Odds ratios, hazard ratios, $95 \%$ confidence intervals, and $\mathrm{p}$ values were calculated. SAS software version 9.4 M3 for Linux was used to perform all data analysis (SAS Institute). This study complies with STROBE guidelines. ${ }^{22}$

\section{Results}

\section{Sample Characteristics}

A total of 57,998 patients in the SEER database met the study criteria and were considered for analysis. Patients were further divided according to tumor behavior and histological classification. Analyses of patient and tumor characteristics were performed within each behavioral group and are represented in Tables 1 and 2 .

Ninety-six percent of the patients $(55,391)$ were diagnosed with benign meningiomas. Patients in this category were predominantly 60-79 years old at diagnosis $(22,314$ [40.3\%]), female $(42,025$ [75.9\%]), and white $(43,041$ [77.7\%]). Tumors were mostly < $20 \mathrm{~mm}(18,802$ [33.9\%]) or $20-40 \mathrm{~mm}(16,721$ [30.2\%]) in size and were predominantly located in the cerebral meninges (46,307 [83.6\%]). One lesion subtype, psammomatous meningioma, had a greater tendency to be located in the spinal meninges (475 [41.2\%]) compared to benign meningiomas in general (2425 [4.4\%]).

Atypical meningiomas were found in 1838 (3.2\%) patients. They mostly occurred in the patients who were female (1086 [59.1\%]), white (1362 [74.1\%]), and ages 40-59 years $(753[41.0 \%])$ or $60-79$ years $(713$ [38.8\%]). The tumors mostly arose in the cerebral meninges (1615 [87.9\%]) and presented with sizes $>40 \mathrm{~mm}$ (1020 [55.5\%]). However, meningiomatosis (NOS) had a more even distribution among the tumor size categories of $<20 \mathrm{~mm}$ (29 [21.8\%]), 20-40 mm (34 [25.6\%]), and > $40 \mathrm{~mm}$ (41 [30.8\%]).

Seven hundred sixty-nine $(1.3 \%)$ cases were identified as anaplastic meningiomas. Much like the other two groups of tumors, the pattern of white (569 [74.0\%]), married (414 [53.8\%]), female (461 [60.0\%]) patients was seen in the anaplastic type. However, the papillary meningioma subtype specifically showed a male predominance (49 [62.8\%]). The age group most affected was 40-59 years old (308 [40.1\%]), followed by 60-79 years old (286 [37.2\%]). Tumors were generally located in the cerebral meninges (602 [78.3\%]). Although most cases had tumors measuring $>40 \mathrm{~mm}$ in size (221 [28.7\%]), a majority of cases in this behavior category had unknown sizes (408 [53.1\%]).

\section{Trends in Treatment Paradigms}

Tables 1 and 2 outline the trends in the treatment para-
TABLE 3. Results of Cox proportional hazards models for benign meningioma

\begin{tabular}{|c|c|c|c|}
\hline Variable & $p$ Value & $\mathrm{HR}$ & $95 \% \mathrm{Cl}$ \\
\hline \multicolumn{4}{|l|}{ Treatment sequence } \\
\hline No treatment & \multicolumn{3}{|c|}{ Reference } \\
\hline Biopsy & 0.0621 & 0.357 & $0.121-1.053$ \\
\hline STR & 0.2992 & 0.521 & $0.152-1.785$ \\
\hline GTR & $0.0129^{*}$ & 0.289 & $0.109-0.769$ \\
\hline RT & 0.5435 & 1.79 & $0.274-11.702$ \\
\hline RT + biopsy & 0.3826 & 0.253 & $0.012-5.529$ \\
\hline $\mathrm{RT}+\mathrm{STR}$ & 0.6517 & 1.395 & $0.329-5.917$ \\
\hline RT + GTR & 0.5371 & 0.608 & $0.125-2.956$ \\
\hline \multicolumn{4}{|l|}{ Histology } \\
\hline Meningioma, NOS & \multicolumn{3}{|c|}{ Reference } \\
\hline Meningothelial meningioma & 0.5486 & 1.277 & $0.574-2.843$ \\
\hline Fibrous meningioma & 0.7147 & 0.726 & $0.131-4.039$ \\
\hline Psammomatous meningioma & 0.4053 & 2.046 & $0.379-11.036$ \\
\hline Angiomatous meningioma & 0.3551 & 2.273 & $0.399-12.95$ \\
\hline Transitional meningioma & 0.7114 & 1.248 & $0.386-4.042$ \\
\hline \multicolumn{4}{|l|}{ Age (yrs) } \\
\hline 0-19 & 0.635 & 2.078 & $0.101-42.6$ \\
\hline $20-39$ & 0.8374 & 1.168 & $0.265-5.14$ \\
\hline $40-59$ & \multicolumn{3}{|c|}{ Reference } \\
\hline $60-79$ & $0.0006^{*}$ & 4.396 & $1.885-10.249$ \\
\hline $80+$ & $<0.0001^{*}$ & 19.123 & $6.941-52.687$ \\
\hline \multicolumn{4}{|l|}{ Race } \\
\hline White & \multicolumn{3}{|c|}{ Reference } \\
\hline Black & $0.0112^{*}$ & 2.612 & $1.244-5.485$ \\
\hline Other & 0.2635 & 1.645 & $0.687-3.937$ \\
\hline \multicolumn{4}{|l|}{ Sex } \\
\hline Male & \multicolumn{3}{|c|}{ Reference } \\
\hline Female & $0.0139 *$ & 0.462 & $0.25-0.855$ \\
\hline \multicolumn{4}{|l|}{ Marital status } \\
\hline Single & \multicolumn{3}{|c|}{ Reference } \\
\hline $\begin{array}{l}\text { Divorced, separated, or } \\
\text { widowed }\end{array}$ & 0.252 & 0.573 & $0.221-1.485$ \\
\hline Married or domestic partner & 0.4601 & 0.742 & $0.337-1.637$ \\
\hline \multicolumn{4}{|l|}{ Anatomical location } \\
\hline Brain meninges & \multicolumn{3}{|c|}{ Reference } \\
\hline Meninges (NOS) & 0.7866 & 1.149 & $0.42-3.141$ \\
\hline Nonmeningeal & 0.1792 & 3.081 & $0.596-15.913$ \\
\hline Spine meninges & 0.5174 & 0.38 & $0.02-7.115$ \\
\hline \multicolumn{4}{|l|}{ Tumor size } \\
\hline$<20 \mathrm{~mm}$ & \multicolumn{3}{|c|}{ Reference } \\
\hline $20-40 \mathrm{~mm}$ & 0.2387 & 2.337 & $0.569-9.598$ \\
\hline$>40 \mathrm{~mm}$ & $0.0383^{*}$ & 4.43 & $1.084-18.114$ \\
\hline \multicolumn{4}{|l|}{ WHO grade } \\
\hline I & \multicolumn{3}{|c|}{ Reference } \\
\hline II & $0.0056^{*}$ & 2.963 & $1.373-6.392$ \\
\hline
\end{tabular}

* Statistically significant. 
TABLE 4. Results of Cox proportional hazards models for atypical meningioma

\begin{tabular}{|c|c|c|c|}
\hline Variable & p Value & $\mathrm{HR}$ & $95 \% \mathrm{Cl}$ \\
\hline \multicolumn{4}{|l|}{ Treatment sequence } \\
\hline GTR & \multicolumn{3}{|c|}{ Reference } \\
\hline No treatment & 0.2437 & 3.107 & $0.462-20.898$ \\
\hline STR & 0.0735 & 2.973 & $0.902-9.801$ \\
\hline RT + biopsy & 0.6189 & 1.600 & $0.251-10.204$ \\
\hline RT + STR & $0.0334^{*}$ & 4.282 & $1.121-16.354$ \\
\hline $\mathrm{RT}+\mathrm{GTR}$ & 0.6284 & 1.353 & $0.398-4.603$ \\
\hline \multicolumn{4}{|l|}{ Histology } \\
\hline Atypical meningioma & \multicolumn{3}{|c|}{ Reference } \\
\hline Meningiomatosis, NOS & 0.3755 & 0.192 & $0.005-7.370$ \\
\hline Clear cell meningioma & 0.3055 & 0.413 & $0.076-2.242$ \\
\hline \multicolumn{4}{|l|}{ Age (yrs) } \\
\hline 0-19 & 0.8308 & 0.705 & $0.029-17.373$ \\
\hline $20-39$ & 0.7527 & 1.247 & $0.316-4.914$ \\
\hline $40-59$ & \multicolumn{3}{|c|}{ Reference } \\
\hline $60-79$ & 0.2786 & 1.784 & $0.626-5.085$ \\
\hline $80+$ & $<0.0001^{*}$ & 45.524 & $11.83-175.178$ \\
\hline \multicolumn{4}{|l|}{ Race } \\
\hline White & \multicolumn{3}{|c|}{ Reference } \\
\hline Black & 0.6821 & 1.269 & $0.406-3.965$ \\
\hline Other & 0.5389 & 0.572 & $0.096-3.398$ \\
\hline \multicolumn{4}{|l|}{ Sex } \\
\hline Male & \multicolumn{3}{|c|}{ Reference } \\
\hline Female & 0.9341 & 1.035 & $0.457-2.344$ \\
\hline \multicolumn{4}{|l|}{ Marital status } \\
\hline Single & \multicolumn{3}{|c|}{ Reference } \\
\hline $\begin{array}{l}\text { Divorced, separated, or } \\
\text { widowed }\end{array}$ & 0.7523 & 0.817 & $0.234-2.858$ \\
\hline Married or domestic partner & 0.2414 & 0.542 & $0.195-1.509$ \\
\hline \multicolumn{4}{|l|}{ Anatomical location } \\
\hline Brain meninges & \multicolumn{3}{|c|}{ Reference } \\
\hline Meninges (NOS) & 0.8688 & 0.859 & $0.141-5.217$ \\
\hline Nonmeningeal & 0.9959 & 1.009 & $0.031-33.056$ \\
\hline Spine meninges & 0.5941 & 2.289 & $0.109-48.141$ \\
\hline \multicolumn{4}{|l|}{ Tumor size } \\
\hline$<20 \mathrm{~mm}$ & \multicolumn{3}{|c|}{ Reference } \\
\hline $20-40 \mathrm{~mm}$ & 0.5415 & 0.378 & $0.017-8.567$ \\
\hline$>40 \mathrm{~mm}$ & 0.9252 & 1.149 & $0.063-20.945$ \\
\hline \multicolumn{4}{|l|}{ WHO grade } \\
\hline II & \multicolumn{3}{|c|}{ Reference } \\
\hline III & 0.0777 & 5.034 & $0.836-30.313$ \\
\hline
\end{tabular}

* Statistically significant.

digms for each type of meningioma. Of the three behavior categories, benign meningiomas were least likely to be treated $(30,291$ [54.7\%]). When they were treated, however, resection was preferred, with GTR being achieved in $13,813(24.9 \%)$ patients. Generally, no radiotherapy $(51,261$ [92.5\%]) was administered. Cases of atypical meningioma were managed mostly by GTR alone (956
TABLE 5. Results of Cox proportional hazards models for anaplastic meningioma

\begin{tabular}{|c|c|c|c|}
\hline Variable & $p$ Value & $\mathrm{HR}$ & $95 \% \mathrm{Cl}$ \\
\hline \multicolumn{4}{|l|}{ Treatment sequence } \\
\hline GTR & \multicolumn{3}{|c|}{ Reference } \\
\hline No treatment & 0.7305 & 1.81 & $0.062-52.894$ \\
\hline Biopsy & 0.0851 & 0.144 & $0.016-1.308$ \\
\hline STR & 0.2441 & 0.259 & $0.027-2.515$ \\
\hline RT + biopsy & 0.0593 & 0.261 & $0.065-1.054$ \\
\hline $\mathrm{RT}+\mathrm{STR}$ & $0.0184^{*}$ & 0.089 & $0.012-0.665$ \\
\hline $\mathrm{RT}+\mathrm{GTR}$ & $0.0019^{*}$ & 0.162 & $0.052-0.511$ \\
\hline \multicolumn{4}{|l|}{ Histology } \\
\hline Meningioma, malignant & \multicolumn{3}{|c|}{ Reference } \\
\hline Papillary meningioma & 0.0880 & 0.273 & $0.061-1.213$ \\
\hline \multicolumn{4}{|l|}{ Age (yrs) } \\
\hline $0-19$ & 0.3120 & 6.486 & $0.173-243.224$ \\
\hline $20-39$ & 0.7937 & 0.659 & $0.029-15.063$ \\
\hline $40-59$ & \multicolumn{3}{|c|}{ Reference } \\
\hline $60-79$ & $0.0113^{*}$ & 4.395 & $1.399-13.808$ \\
\hline $80+$ & 0.0640 & 4.570 & $0.915-22.822$ \\
\hline \multicolumn{4}{|l|}{ Race } \\
\hline White & \multicolumn{3}{|c|}{ Reference } \\
\hline Black & 0.4161 & 1.650 & $0.494-5.514$ \\
\hline Other & 0.9532 & 1.034 & $0.337-3.175$ \\
\hline \multicolumn{4}{|l|}{ Sex } \\
\hline Male & \multicolumn{3}{|c|}{ Reference } \\
\hline Female & 0.5479 & 1.295 & $0.557-3.014$ \\
\hline \multicolumn{4}{|l|}{ Marital status } \\
\hline Single & \multicolumn{3}{|c|}{ Reference } \\
\hline $\begin{array}{l}\text { Divorced, separated, or } \\
\text { widowed }\end{array}$ & $0.0484^{*}$ & 8.001 & $1.014-63.108$ \\
\hline Married or domestic partner & $0.0498^{*}$ & 7.463 & $1.002-55.603$ \\
\hline \multicolumn{4}{|l|}{ Anatomical location } \\
\hline Brain meninges & \multicolumn{3}{|c|}{ Reference } \\
\hline Meninges (NOS) & 0.6773 & 1.343 & $0.335-5.38$ \\
\hline Nonmeningeal & 0.9859 & 1.033 & $0.027-39.155$ \\
\hline Spine meninges & 0.9797 & 0.957 & $0.031-29.142$ \\
\hline \multicolumn{4}{|l|}{ Tumor size } \\
\hline$<20 \mathrm{~mm}$ & \multicolumn{3}{|c|}{ Reference } \\
\hline $20-40 \mathrm{~mm}$ & 0.5001 & 0.448 & $0.043-4.629$ \\
\hline$>40 \mathrm{~mm}$ & 0.8490 & 1.213 & $0.167-8.819$ \\
\hline \multicolumn{4}{|l|}{ WHO grade } \\
\hline II & \multicolumn{3}{|c|}{ Reference } \\
\hline III & $0.0050^{*}$ & 8.625 & $1.914-38.862$ \\
\hline
\end{tabular}

* Statistically significant.

[52.0\%]), followed by GTR and radiation (306 [16.6\%]). A total of $535(29.1 \%)$ patients did receive radiotherapy. Anaplastic tumors were managed similarly to atypical tumors, with most treated via GTR alone (258 [33.6\%]) or GTR followed by radiation (151 [19.6\%]). A higher proportion of patients with anaplastic tumors received radiotherapy (267 [34.7\%]) compared to the other groups. 
TABLE 6. Treatment sequence results of Cox proportional hazards models for atypical meningioma with STR as the baseline treatment*

\begin{tabular}{crcc}
\hline Treatment Sequence & $\mathrm{p}$ Value & $\mathrm{HR}$ & $95 \% \mathrm{Cl}$ \\
\hline STR & Reference & & \\
\hline No treatment & 0.9671 & 1.045 & $0.128-8.562$ \\
\hline GTR & 0.0735 & 0.336 & $0.102-1.109$ \\
\hline RT + biopsy & 0.5530 & 0.538 & $0.070-4.165$ \\
\hline RT + STR & 0.6438 & 1.440 & $0.307-6.766$ \\
\hline RT + GTR & 0.3012 & 0.455 & $0.102-2.024$ \\
\hline
\end{tabular}

* All other findings for histology, age, sex, etc. from Table 4 are unchanged in this analysis.

\section{Multivariate Results}

The outcomes of different treatment sequences were examined using multivariate analysis with the Cox proportional hazards model, controlling for histological characteristics, demographic factors, anatomical location and size of the tumor, and WHO grade. Tumors without a known WHO grade were excluded from this analysis. In the benign meningioma group (11,673 cases; Table 3), GTR, as compared to no treatment, yielded a significant decrease in the causespecific mortality risk (HR 0.289, 95\% CI 0.109-0.769, p = 0.0129 ). In the analysis of atypical and anaplastic meningiomas, the reference group was set as GTR in order to better examine the effects of radiotherapy on the risk of death since all standard of care in these treatments involved attempts at GTR. In doing this, we observed that for anaplastic tumors (166 cases; Table 5), adjuvant radiation led to a significant decrease in mortality for the patients who had undergone either GTR (HR 0.162, 95\% CI 0.052-0.511, $\mathrm{p}=0.0019$ ) or
STR (HR 0.089, 95\% CI 0.012-0.665, p = 0.0184). However, for atypical tumors (1092 cases; Table 4), adjuvant radiation therapy with STR increased the mortality risk compared to GTR alone (HR 4.282, 95\% CI 1.121-16.354, $\mathrm{p}=0.0334$ ). Moreover, we saw that the addition of adjuvant radiotherapy had no statistically significant effect in patients with GTR (HR 1.353, 95\% CI 0.398-4.603, $\mathrm{p}=0.6284$ ).

Across all behavior types of meningioma, an increase in mortality risk was seen in the patient populations ages 60-79 and above 80 compared to patients $40-59$ years old (HR 4.396, $\mathrm{p}=0.0006$ and HR 19.123, $\mathrm{p}<0.0001$ for benign; HR 1.784, $p=0.2786$ and HR 45.523, $p<0.0001$ for atypical; HR 4.395, $\mathrm{p}=0.0113$ and HR 4.570, $\mathrm{p}=0.0640$ for anaplastic). In benign meningiomas, significantly increased mortality was associated with an increased tumor size and WHO grade II tumors (HR 4.430, 95\% CI 1.084-18.114, $\mathrm{p}=0.0383$; HR 2.963, 95\% CI 1.373-6.392, $\mathrm{p}=0.0056$, respectively). A similar trend was seen for anaplastic tumors, in which WHO grade III meningiomas had unfavorable outcomes compared to WHO grade II tumors (HR 8.625, 95\% CI 1.914-38.862, $\mathrm{p}=0.005$ ).

In order to more closely examine the increased mortality risk associated with both STR and RT in atypical meningiomas, we performed a breakaway analysis comparing all treatment groups, with STR as the reference group (Table 6). We found that adjuvant radiation did not significantly lower risks in the setting of performing STR (HR 1.440, 95\% CI 0.307-6.766, $\mathrm{p}=0.6438$ ).

Graphic analysis using the Kaplan-Meier curve was performed to evaluate the association between treatment sequences involving resection or radiation and survival outcomes in atypical meningiomas (Fig. 1). Using a logrank test with Sidak correction, we found no significant differences between any of the four treatment groups tested.

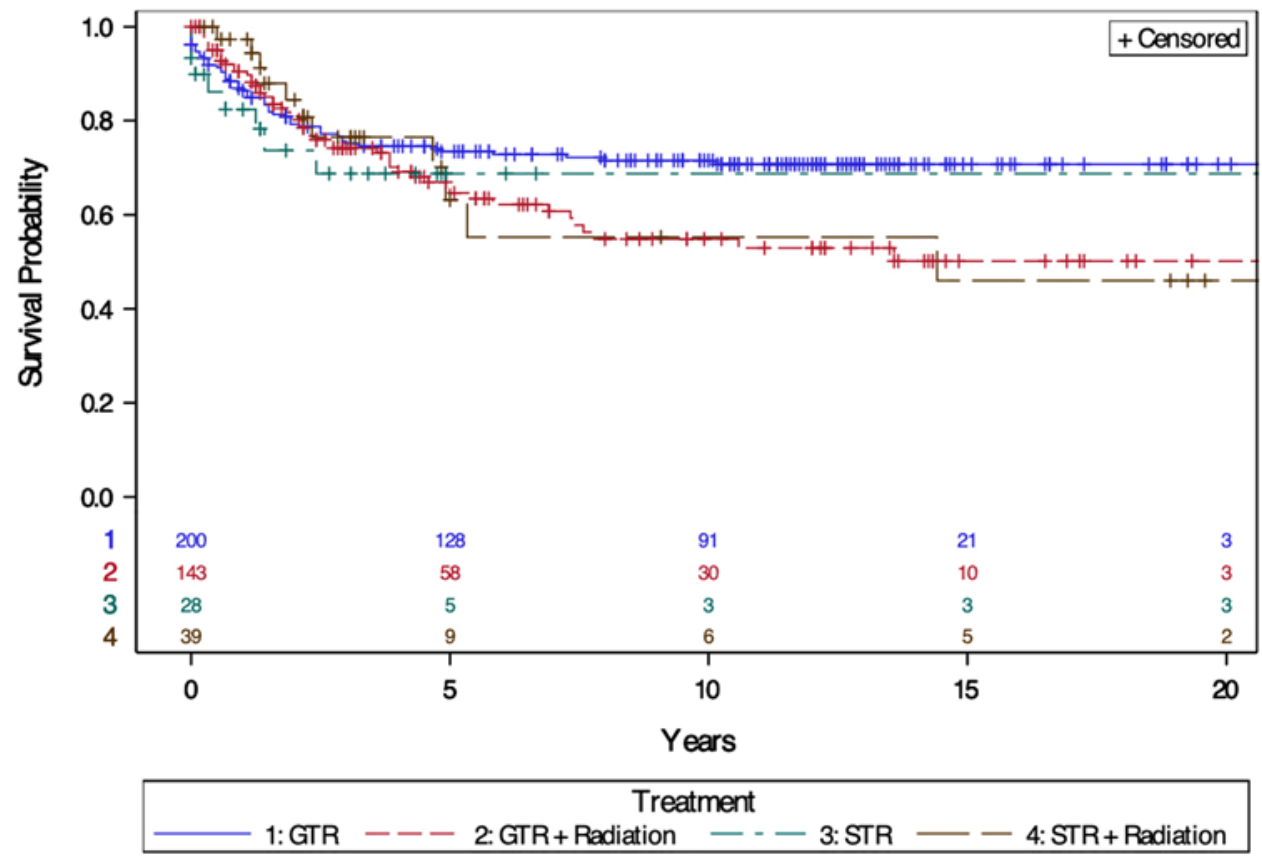

FIG. 1. Kaplan-Meier survival curve assessing cause-specific mortality for patients with atypical meningiomas. No statistically significant differences were noted between treatment groups. 


\section{Discussion}

This largest-to-date SEER-based assessment of the epidemiology of primary meningiomas included a total of 57,998 patients. Our study confirmed previously reported information suggesting a predisposition for benign meningiomas in older, female, white populations ${ }^{1,5,10}$ with the tumors predominantly smaller than $40 \mathrm{~mm}$ and located in the cerebral meninges. ${ }^{1}$ Similar distributions of patient demographics were seen in the atypical and anaplastic cases with the only difference in lesion characteristics being a larger proportion of cases with tumors larger than $40 \mathrm{~mm}$, which is consistent with the literature. ${ }^{2}$

The Cox proportional hazards model allowed us to assess the relative hazard of various treatment paradigms on the cause-specific mortality of patients with various levels of primary meningioma. For benign meningiomas, standard of care is a "wait and watch" approach for most of the nonaggressively growing tumors, performing resection only in those cases in which size may create a mass effect or growth rates increase. ${ }^{4}$ This is reflected in our analysis, which showed that the majority of patients received no treatment and that even when resection was performed, radiotherapy was uncommon. Our analysis also showed a significantly lower HR for patients who underwent GTR but no significant HRs for the other treatments. This suggests that in patients with symptoms associated with their benign tumors, resection without the use of adjuvant radiotherapy is beneficial, which is consistent with the literature. ${ }^{5}$

In atypical meningioma, the standard of care is GTR when possible, although there is no consistency regarding the role of radiotherapy. ${ }^{2,3,19}$ Our analysis showed that more than a quarter of all atypical meningiomas were treated with adjuvant radiotherapy. In cases in which STR was performed with adjuvant radiotherapy, there was a significantly increased hazard as compared to GTR alone. This finding confirms that GTR should always be attempted. However, our data showed that after GTR, radiation did not impart a significant survival benefit despite the trend of using adjuvant radiotherapy for these tumors. Additionally, our findings showed that, compared to a baseline of performing STR only, radiotherapy following STR was not associated with a lower HR. Therefore, even in cases in which surgeons are unable to resect the entirety of an atypical meningioma, our data do not support the use of concomitant radiotherapy.

The standard of care for anaplastic meningioma is to perform GTR if possible and to provide adjuvant radiotherapy. ${ }^{16}$ Our data revealed that only about a third of all patients received adjuvant radiotherapy despite this standard. Our analysis showed that, compared to GTR alone, patients undergoing both GTR and adjuvant radiotherapy or both STR and adjuvant radiotherapy had significantly lower HRs. Prior retrospective research similarly showed that the use of all available treatment methods in anaplastic populations is associated with an increased 10-year survival. ${ }^{20}$ This supports the current standard of including adjuvant radiotherapy in the treatment sequence, regardless of the degree of resection, to improve patient outcomes.

Because of the limitations inherent to the SEER database, the results of this analysis should be taken with a degree of caution. Primarily, the lack of NF2 status in- formation for patients in the database precludes one from extrapolating on a known driver of meningioma. ${ }^{17}$ Without the patient-level NF2 status, there may be a level of stratification of treatment choices for the patients with atypical meningiomas that is not reflected in the cofactors controlled for in the analysis. Additionally, the SEER database is limited in that it does not offer data on the dosing and modality of the radiation provided; therefore, we could not make any inferences about the relative magnitude of radiation therapy received by any one patient population or the overarching trends in the type of radiation therapy given over time. This absent characterization of radiation is an important consideration given that patients who are clinically judged to have a high risk of recurrence may receive a different level or modality of radiation than the general population. . Additionally, we were unable to assess whether certain types of radiation therapy or dosing regimens were associated with increased rates of survival.

Neither were there any Simpson resection grades in this data set, which would have provided for a more graded evaluation of the level of resection. While this grading scheme is often used by surgeons to predict the probability of recurrence, our analysis was limited to delineating GTR versus STR. These factors impact the susceptibility of recurrence, which is another limitation in using this type of registry data since it is not possible to track the length of progression-free survival or whether a particular patient had a meningioma recurrence. Instead, the metric of causespecific mortality is the only outcome available. Previous work has shown that radiation may be helpful in preventing further recurrence of meningioma, ${ }^{9}$ but the evidence is not definitive. ${ }^{6}$ Although our study showed that the use of radiation in patients with atypical meningiomas may not confer a survival benefit in patients with single tumors, we cannot determine whether radiation prevents recurrent tumors.

One factor that may not provide a clear bias but is worth noting is the large proportion of patients in the SEER database who did not have WHO grading of their tumors. These patients were removed from the survival analysis, and this decision could have introduced confounding if the lack of documentation is associated with prognostic factors. However, it is reasonable to assume that whether a physician noted a WHO grade for a particular patient in the registry is independent of the disease severity. Additionally, it should be acknowledged that using registry databases such as SEER comes with inherent limitations such as bias based on locations in the country that contribute to the database and the potential differences in reporting methodologies at each location. Registry databases may also underreport cases for which there are no clinical symptoms and therefore present with selection bias. ${ }^{13}$

In the absence of a similarly large-scale database that includes these other factors, this analysis represents the largest available baseline from which to work, with acknowledgment of its limitations, and makes valuable suggestions about the treatment choices that physicians make. Moreover, given the limitations of using this type of registry data, we strongly suggest that further work be done in a prospective manner (while documenting a wide variety of clinically relevant cofactors) to assess the effectiveness of adjuvant radiotherapy use (while noting the specific type 
and dosing regimen) after resection of an atypical meningioma.

\section{Conclusions}

The results of this retrospective population-based study provide some insight into the role of adjuvant radiotherapy in the management of meningiomas. We demonstrated that the use of radiation after an attempt at GTR did not impart a mortality risk reduction in cases of benign or atypical meningiomas. Notably, adjuvant radiation did not significantly reduce the hazard risk in the STR of atypical meningiomas, suggesting that radiotherapy may not be beneficial despite residual tumor. On the other hand, anaplastic meningiomas did see significant risk reduction with radiotherapy following both GTR and STR. Given the inherent limitations of the SEER database and retrospective analysis, our results are insufficient to redefine the standard management of these tumors; however, they do provide a direction for prospective randomized clinical trials to measure the outcomes of radiation therapy after meningioma resection and define which populations may benefit from adjuvant radiotherapy.

\section{References}

1. Agarwal V, McCutcheon BA, Hughes JD, Carlson ML, Glasgow AE, Habermann EB, et al: Trends in management of intracranial meningiomas: analysis of 49,921 cases from modern cohort. World Neurosurg 106:145-151, 2017

2. Aizer AA, Bi WL, Kandola MS, Lee EQ, Nayak L, Rinne ML, et al: Extent of resection and overall survival for patients with atypical and malignant meningioma. Cancer 121:4376-4381, 2015

3. Barthélemy E, Loewenstern J, Konuthula N, Pain M, Hall J, Govindaraj S, et al: Primary management of atypical meningioma: treatment patterns and survival outcomes by patient age. J Cancer Res Clin Oncol 144:969-978, 2018

4. Buerki RA, Horbinski CM, Kruser T, Horowitz PM, James CD, Lukas RV: An overview of meningiomas. Future Oncol 14:2161-2177, 2018

5. Cahill KS, Claus EB: Treatment and survival of patients with nonmalignant intracranial meningioma: results from the Surveillance, Epidemiology, and End Results Program of the National Cancer Institute. Clinical article. J Neurosurg 115:259-267, 2011

6. Champeaux C, Houston D, Dunn L: Atypical meningioma. A study on recurrence and disease-specific survival. Neurochirurgie 63:273-281, 2017

7. Chan AW, Bernstein KD, Adams JA, Parambi RJ, Loeffler JS: Dose escalation with proton radiation therapy for high-grade meningiomas. Technol Cancer Res Treat 11:607-614, 2012

8. Dudley RWR, Torok MR, Randall S, Béland B, Handler MH, Mulcahy-Levy JM, et al: Pediatric versus adult meningioma: comparison of epidemiology, treatments, and outcomes using the Surveillance, Epidemiology, and End Results database. J Neurooncol 137:621-629, 2018

9. Dziuk TW, Woo S, Butler EB, Thornby J, Grossman R, Dennis WS, et al: Malignant meningioma: an indication for initia aggressive surgery and adjuvant radiotherapy. J Neurooncol 37:177-188, 1998

10. Garzon-Muvdi T, Yang W, Lim M, Brem H, Huang J: Atypical and anaplastic meningioma: outcomes in a population based study. J Neurooncol 133:321-330, 2017

11. Grossbach AJ, Mahaney KB, Menezes AH: Pediatric meningiomas: 65-year experience at a single institution. J Neurosurg Pediatr 20:42-50, 2017

12. Heinze G: A comparative investigation of methods for logis- tic regression with separated or nearly separated data. Stat Med 25:4216-4226, 2006

13. Karhade AV, Larsen AMG, Cote DJ, Dubois HM, Smith TR: National databases for neurosurgical outcomes research: options, strengths, and limitations. Neurosurgery 83:333-344, 2018

14. Kotecha RS, Junckerstorff RC, Lee S, Cole CH, Gottardo NG: Pediatric meningioma: current approaches and future direction. J Neurooncol 104:1-10, 2011

15. Ostrom QT, Gittleman H, Fulop J, Liu M, Blanda R, Kromer C, et al: CBTRUS Statistical Report: Primary Brain and Central Nervous System Tumors Diagnosed in the United States in 2008-2012. Neuro Oncol 17 (Suppl 4):iv1-iv62, 2015

16. Paldor I, Awad M, Sufaro YZ, Kaye AH, Shoshan Y: Review of controversies in management of non-benign meningioma. J Clin Neurosci 31:37-46, 2016

17. Rogers L, Barani I, Chamberlain M, Kaley TJ, McDermott M, Raizer J, et al: Meningiomas: knowledge base, treatment outcomes, and uncertainties. A RANO review. J Neurosurg 122:4-23, 2015

18. Solheim O, Torsteinsen M, Johannesen TB, Jakola AS: Effects of cerebral magnetic resonance imaging in outpatients on observed incidence of intracranial tumors and patient survival: a national observational study. J Neurosurg 120:827-832, 2014

19. Stessin AM, Schwartz A, Judanin G, Pannullo SC, Boockvar JA, Schwartz TH, et al: Does adjuvant external-beam radiotherapy improve outcomes for nonbenign meningiomas? A Surveillance, Epidemiology, and End Results (SEER)-based analysis. J Neurosurg 117:669-675, 2012

20. Sughrue ME, Sanai N, Shangari G, Parsa AT, Berger MS, McDermott MW: Outcome and survival following primary and repeat surgery for World Health Organization Grade III meningiomas. J Neurosurg 113:202-209, 2010

21. Vernooij MW, Ikram MA, Tanghe HL, Vincent AJ, Hofman A, Krestin GP, et al: Incidental findings on brain MRI in the general population. N Engl J Med 357:1821-1828, 2007

22. von Elm E, Altman DG, Egger M, Pocock SJ, Gøtzsche PC, Vandenbroucke JP: The Strengthening the Reporting of Observational Studies in Epidemiology (STROBE) statement: guidelines for reporting observational studies. J Clin Epidemiol 61:344-349, 2008

23. Westwick HJ, Shamji MF: Effects of sex on the incidence and prognosis of spinal meningiomas: a Surveillance, Epidemiology, and End Results study. J Neurosurg Spine 23:368-373, 2015

24. Yano S, Kuratsu J: Indications for surgery in patients with asymptomatic meningiomas based on an extensive experience. J Neurosurg 105:538-543, 2006

\section{Disclosures}

Dr. Mehta has been a consultant for DePuy Synthes and Globus Medical.

\section{Author Contributions}

Conception and design: Mehta, Reddy, Ryoo, Denyer. Acquisition of data: Mehta. Analysis and interpretation of data: Reddy, Ryoo, Denyer, McGuire. Drafting the article: Reddy, Ryoo, Denyer. Critically revising the article: Ryoo, McGuire. Reviewed submitted version of manuscript: all authors. Statistical analysis: Reddy, Denyer. Study supervision: Mehta.

\section{Supplemental Information \\ Videos}

Video Abstract. https://vimeo.com/334430572.

\section{Correspondence}

Ankit I. Mehta: University of Illinois at Chicago, Chicago, IL. ankitm@uic.edu. 